\title{
COMMUTATORS WITH POWERS OF AN UNBOUNDED OPERATOR IN HILBERT SPACE
}

\author{
FELIX E. BROWDER
}

Let $H$ be a Hilbert space, $B$ a bounded linear operator on $H, A$ a closed, densely defined, unbounded positive self-adjoint linear operator in $H$. It is the object of the present paper to prove the following theorem:

THEOREM. Suppose that for a given positive integer $n$,

$$
\begin{aligned}
B A^{n}-A^{n} B & \subseteq B_{1} A^{n-1}, \\
B_{1} A^{n}-A^{n} B_{1} & \subseteq B_{2} A^{n-1},
\end{aligned}
$$

where $B_{1}$ and $B_{2}$ are bounded linear operators on $H$. Then $B A-A B$ is bounded.

The original context in which this theorem arose was one in which $A^{2}$ was the differential operator $(I-\Delta)$ on a compact Riemannian manifold $M, B$ a singular elliptic operator on $M, n=2$. The theorem was proved by the writer in connection with a program begun by R. Palais for giving an intrinsic treatment of singular integral operators on manifolds without the use of localization arguments. We publish it here since it may have other interesting applications.

LEMMA 1. There exists a constant $c_{n}$ such that for $u$ in $D(A)$,

$$
A u=c_{n} \int_{0}^{\infty} t^{1 / n}\left\{\left(A^{n}+i t I\right)^{-1} u+\left(A^{n}-i t I\right)^{-1} u\right\} d t .
$$

Proof of Lemma 1. Let $E(\lambda)$ be the spectral family of the positive self-adjoint operator $A$. Then:

$$
\begin{aligned}
\int_{0}^{\infty} t^{1 / n}\left\{\left(A^{n}+i t I\right)^{-1}\right. & \left.+\left(A^{n}-i t I\right)^{-1}\right\} d t \\
& =\int_{0}^{\infty} t^{1 / n} \int_{\lambda \Sigma \lambda_{0}>0} 2 \lambda^{n}\left(\lambda^{2 n}+t^{2}\right)^{-1} d E(\lambda) d t \\
& =\int_{\lambda \Sigma \lambda_{0}} 2 \lambda^{n} \int_{0}^{\infty} t^{1 / n}\left(\lambda^{2 n}+t^{2}\right)^{-1} d t d E(\lambda) \\
& =\int_{\lambda \Sigma \lambda_{0}} 2 \lambda\left(\int_{0}^{\infty} t^{1 / n}\left(1+t^{2}\right)^{-1} d t\right) d E(\lambda)=c_{n}^{-1} A .
\end{aligned}
$$

Received by the editors January 13, 1965. 
Proof of the Theorem. For each $t \geqq 0$, we have

$$
B\left(A^{n} \pm i t I\right)-\left(A^{n} \pm i t I\right) B \subseteq B_{1} A^{n-1} \text {. }
$$

Multiplying on both sides by $\left(A^{n} \pm i t I\right)^{-1}$, we obtain

(6) $\left(A^{n} \pm i t I\right)^{-1} B-B\left(A^{n} \pm i t I\right)^{-1} \subseteq\left(A^{n} \pm i t I\right)^{-1} B_{1} A^{n-1}\left(A^{n} \pm i t I\right)^{-1}$.

\section{Similarly}

$$
\begin{aligned}
\left(A^{n} \pm i t I\right)^{-1} B_{1}-B_{1}\left(A^{n} \pm i t I\right)^{-1} & \\
& \subseteq\left(A^{n} \pm i t I\right)^{-1} B_{2} A^{n-1}\left(A^{n} \pm i t I\right)^{-1}
\end{aligned}
$$

By the representation (4) for $A$ and equation (6), we have

$$
\begin{aligned}
B A-A B \subseteq-c_{n} \int_{0}^{\infty} t^{1 / n} & \left\{\left(A^{n}+i t I\right)^{-1} B_{1} A^{n-1}\left(A^{n}+i t I\right)^{-1}\right. \\
& \left.+\left(A^{n}-i t I\right)^{-1} B_{1} A^{n-1}\left(A^{n}-i t I\right)^{-1}\right\} d t
\end{aligned}
$$

Applying equation (7), we see that

$$
B A-A B \subseteq-c_{n}\left(I_{1}+I_{2}\right) \text {, }
$$

where

$$
\begin{aligned}
I_{1}=B_{1} \int_{0}^{\infty} t^{1 / n}\left\{\left(A^{n}+i t I\right)^{-1} A^{n-1}(\right. & \left.A^{n}+i t I\right)^{-1} \\
& \left.+\left(A^{n}-i t I\right)^{-1} A^{n-1}\left(A^{n}-i t I\right)^{-1}\right\} d t
\end{aligned}
$$

and

$$
\begin{aligned}
I_{2}=\int_{0}^{\infty} t^{1 / n}\{ & \left(A^{n}+i t I\right)^{-1} B_{2} A^{n-1}\left(A^{n}+i t I\right)^{-1} A^{n-1}\left(A^{n}+i t I\right)^{-1} \\
& \left.+\left(A^{n}-i t I\right)^{-1} B_{2} A^{n-1}\left(A^{n}-i t I\right)^{-1} A^{n-1}\left(A^{n}-i t I\right)^{-1}\right\} d t .
\end{aligned}
$$

For $I_{1}$, we have

$$
\begin{aligned}
I_{1} & =B_{1} \int_{0}^{\infty} t^{1 / n} \int_{\lambda \geq \lambda_{0}}\left\{\lambda^{n-1}\left(\lambda^{n}+i t\right)^{-2}+\lambda^{n-1}\left(\lambda^{n}-i t\right)^{-2}\right\} d E(\lambda) d t \\
& =B_{1} \int_{\lambda \geq \lambda_{0}} \int_{0}^{\infty} t^{1 / n} \lambda^{n-1}\left\{\left(\lambda^{n}+i t\right)^{-2}+\left(\lambda^{n}-i t\right)^{-2}\right\} d t d E(\lambda) \\
& =B_{1} \int_{\lambda \geq \lambda_{0}} \lambda^{n-1} \int_{0}^{\infty} t^{1 / n}\left(2 \lambda^{2}-2 t^{2}\right)\left(\lambda^{2 n}+t^{2}\right)^{-2} d t d E(\lambda) \\
& =B_{1} \int_{\lambda \geq \lambda_{0}}\left\{\int_{0}^{\infty} 2 s^{1 / n}\left(1-s^{2}\right)\left(1+s^{2}\right)^{-2} d s\right\} d E(\lambda)=c B_{1} .
\end{aligned}
$$


For $I_{2}$, we apply the simple estimates

$$
\begin{aligned}
\left\|A^{n-1}\left(A^{n} \pm i t I\right)^{-1}\right\| & \leqq c(c+t)^{-1 / n} \\
\left\|\left(A^{n} \pm i t I\right)^{-1}\right\| & \leqq(c+t)^{-1}
\end{aligned}
$$

for a given $c>0$, and obtain

$\left\|\left(A^{n} \pm i t I\right)^{-1} B_{2} A^{n-1}\left(A^{n} \pm i t I\right)^{-1} A^{n-1}\left(A^{n} \pm i t I\right)^{-1}\right\| \leqq c_{1}(c+t)^{-1-2 / n}$.

Hence,

$$
\left\|I_{2}\right\| \leqq 2 c_{1} \int_{0}^{\infty} t^{1 / n}(c+t)^{-1-2 / n} d t<+\infty .
$$

Since both $I_{1}$ and $I_{2}$ are bounded, so is $B A-A B$. q.e.d.

An obvious modification of the above proof yields the following more general result:

Theorem 2. Let $A$ be a positive self-adjoint operator, $B$ a bounded operator on $H$, and suppose that there exist nine bounded operators $B_{1}, \cdots, B_{9}$ on $H$ such that

$$
\begin{aligned}
& B A^{n}-A^{n} B \subseteq B_{1} A^{n-1}+A^{n-1} B_{2}+B_{3}, \\
& B_{1} A^{n}-A^{n} B_{1} \subseteq B_{4} A^{n-1}+A^{n-1} B_{5}+B_{6}, \\
& B_{2} A^{n}-A^{n} B_{2} \subseteq B_{7} A^{n-1}+A^{n-1} B_{8}+B_{9} .
\end{aligned}
$$

Then $B A-A B$ is bounded.

More generally still, we can include on the right of equations (9), (10), and (11) terms of the form $\sum_{j<n-1} B_{j}^{\prime} A^{j}+A^{i} B_{j}^{\prime \prime}$. (This actually follows from the statement of Theorem 2.) By use of the functional calculus for closed operators with appropriate existence and growth conditions on resolvents, we can remove the self-adjointness assumption on $A$ and carry over the result to Banach spaces (with a rather unwieldy hypothesis on the operator $A$ ).

University of Chicago 